\title{
EVALUACIÓN DE LA AMENAZA Y CAUSAS DE INUNDACIÓN EN LA CIUDAD DE TURRIALBA, COSTA RICA
}

\author{
Elena Badilla ${ }^{1}$, Cees J. van Westen ${ }^{2} \&$ Nanette C. Kingma ${ }^{2}$ \\ ${ }^{1}$ Escuela Centroamericana de Geología, Universidad de Costa Rica \\ E-mail: ebadilla@geologia.ucr.ac.cr \\ ${ }^{2}$ International Institute for Geo-Information Science and Earth Observation, The \\ Netherlands, E-mail: westen@itc.nl, kingma@itc.nl
}

(Recibido: 21/8/02; Aceptado: 6/1/03)

\begin{abstract}
Turrialba City has been frequently affected by flash floods of Colorado River, Gamboa Stream and tributaries. These events are caused by the combination of meteorological conditions, geomorphological factors and the uncontrolled urban expansion along these rivers.

A geomorphological description of the study area is provided, mainly on the basis of photo-interpretation and field observations. Subsequently, a different degree of flood hazard is assigned to each geomorphological unit, depending on their physical characteristics, on the existing urban features and large scale topographic elements, and on the occurrence and behaviour of similar events in the past. In this way it is demonstrated that Turrialba City is located in an area with high to medium flood hazard.

Finally, the riverbank erosion problem is described. This phenomena is currently becoming more important, specially along the Turrialba River.
\end{abstract}

RESUMEN: La ciudad de Turrialba es frecuentemente afectada por inundaciones relámpago del río Colorado, la quebrada Gamboa y sus afluentes. Estos eventos son causados por la combinación de condiciones meteorológicas, factores geomorfológicos y el descontrolado crecimiento urbano a lo largo de los cauces de los ríos.

El área de estudio se describe desde un punto de vista geomorfológico, con base en fotointerpretación y observaciones de campo. Luego, un grado diferente de amenaza de inundación es asignado a cada unidad geomorfológica, dependiendo de sus características físicas, de las condiciones urbanas y elementos de microrelieve existentes, y de la ocurrencia y comportamiento de eventos similares en el pasado. De esta manera se establece que la ciudad de Turrialba se ubica dentro de áreas con una amenaza de inundación alta a media. Por último, se describe la problemática de erosión fluvial, fenómeno que ha ido cobrando más importancia en los últimos años, especialmente a lo largo del río Turrialba.

\section{INTRODUCCIÓN}

La ciudad de Turrialba es frecuentemente afectada por inundaciones relámpago de los pequeños ríos que bajan de las montañas ubicadas al W y SW (río Colorado, quebradas pequeños cursos de agua). El sector históricamente más afectado ha sido siempre el centro de la ciudad. 
Los grandes ríos de la zona (Turrialba, Aquiares y Azul) no han causado inundaciones al menos desde 1891. Esto se debe a que poseen una mayor capacidad de descarga. A pesar de esto, una intensa erosión se ha dado y se sigue dando a lo largo de sus bancos.

El objetivo principal del estudio es evaluar la amenaza de inundación y sus causas con base en las características geomorfológicas, climatológicas y urbanas del área, complementadas con la escasa información disponible relacionada con la ocurrencia y las características de eventos históricos. Este método de evaluación representa una alternativa en áreas donde, por escasez de datos, se hace imposible llevar a cabo estudios estadísticos o hidrológicos y modelaciones hidráulicas.

El procedimiento seguido incluye básicamente cuatro etapas: trabajo de campo, revisión bibliográfica, interpretación de fotografías aéreas e imágenes de satélite $y$, finalmente, la evaluación de la amenaza y causas de inundación, incluyendo la creación del mapa de amenaza de inundación del área de estudio mediante la aplicación de SIG (Sistemas de Información Geográfica).

Durante el trabajo de campo se recopiló información sobre eventos históricos y sus características, sobre algunos aspectos geomorfológicos de detalle y especialmente sobre la distribución y el crecimiento urbano y su relación con el comportamiento de las aguas dentro de la ciudad durante las crecidas. La revisión bibliográfica se centró en la búsqueda de información sobre uso del suelo, eventos históricos y estudios hidrológicos en la zona.

A partir de la interpretación de fotografías aéreas a diferentes escalas (1:40 000 de 1998, 1:20 000 de 1988 y 1:5000 de fecha desconocida) y una imagen tipo anaglifo escala 1:40 000 (construida a partir de banda pancromática de Landsat TM de enero 2001), se extrajo información que posteriormente fue utilizada en la determinación de las causas de inundación y la creación del mapa geomorfológico.

\section{CARACTERÍSTICAS DE LA CUENCA DEL RÍO TURRIALBA}

La cuenca del río Turrialba se localiza en la provincia de Cartago, parte central de Costa Rica. Está delimitada por las coordenadas Lambert Costa Rica 207162-222423 N y 555076574629 E (Fig. 1), de las hojas topográficas Tucurrique e Istarú escala 1:50 000 del Instituto Geográfico Nacional (IGN).

La cuenca ha sido subdividida por Cardona et al. (2000) en 5 subcuencas, cuyas características se muestran en el Cuadro 1.

El clima en la zona es muy complejo, porque es influenciado por variables micro-climáticas. Estas condiciones meteorológicas ocasionan fuertes precipitaciones relacionadas con los vientos húmedos provenientes del Caribe a través del cañón del río Reventazón. Por lo general, las lluvias se dan en la cuenca media, sólo bajo condiciones especiales (huracanes, depresiones, etc.), donde las lluvias pueden alcanzar la cuenca superior. La precipitación promedio anual para toda la cuenca es de $2289,5 \mathrm{~mm}$; se incrementa desde las cuencas superior e inferior hacia la cuenca media, con un pico en el poblado de Aquiares, debido a la distribución de los vientos húmedos (Calvo, 1993).

Morfológicamente, la cuenca del río Turrialba se caracteriza por laderas de pendiente pronunciada y cambios abruptos de pendiente. El río Turrialba corre, en sólo $17,2 \mathrm{~km}$, desde las faldas del volcán Turrialba a 2500 metros sobre

Cuadro 1

Subdivisión y características de la cuenca Turrialba (Cardona et al., 2000)

\begin{tabular}{lrrrr}
\hline Nombre & Área $(\mathrm{km} 2)$ & \multicolumn{2}{c}{ Elevación (m) } & \multicolumn{2}{c}{ Longitud } \\
& & Máxima & Mínima & del río (m) \\
\hline Turrialba & 63.65 & 2500 & 640 & 17200 \\
Colorado & 10.38 & 1220 & 645 & 6700 \\
Gamboa & 1.67 & 960 & 620 & 2500 \\
Aquiares & 22.93 & 2500 & 635 & 11300 \\
Azul & 4.76 & 1040 & 600 & 3800 \\
\hline
\end{tabular}


el nivel del mar, hasta las planicies aluviales ubicadas a una altitud de $640 \mathrm{~m}$ (Cardona et al., 2000). Esto implica el paso de pendientes de 40$60 \%$ en la cuenca alta a pendientes de $3-4 \%$ en la parte baja, donde se encuentra la ciudad de Turrialba (Solís et al., 1994).

El río Turrialba tiene un curso muy dinámico con cambios horizontales y verticales, de acuerdo con la necesidad de adaptar su perfil de equilibrio a las variables condiciones hidrológicas de la cuenca (García, 1990).

\section{DEFINICIÓN DEL ÁREA DE ESTUDIO}

Este estudio se enfoca en la ciudad de Turrialba, localizada en la parte baja de la cuenca del río Turrialba, en las coordenadas $9^{\circ} 54^{\prime} 24^{\prime \prime} \mathrm{N}$ y $83^{\circ} 41^{\prime} 06^{\prime \prime} \mathrm{W}$, a $640 \mathrm{msnm}$. El área de estudio corresponde con un rectángulo de unos $16 \mathrm{~km}^{2}$, delimitado por las coordenadas Lambert Costa Rica 208320-211950 N y 569680-573770 E de la hoja topográfica Tucurrique (Fig. 1).

Los ríos encontrados en el área son:
Turrialba, Aquiares, Azul y Colorado, así como la quebrada Gamboa y su afluente Túnel. Además, las quebradas Poró, Puente Vigas y Barahona, afluentes del Colorado (Fig. 2).

\section{MÉTODO PROPUESTO PARA LA EVALUACIÓN DE AMENAZA DE INUNDACIÓN}

Una amenaza natural es la probabilidad de ocurrencia, dentro de un período de tiempo determinado y un área dada, de un fenómeno potencialmente dañino (UNDRO, 1983). Es un evento físico que tiene un impacto en los seres humanos y su ambiente y, a menos que esta combinación se dé (evento-población), no habrá daño o desastre (Alexander, 1993).

Esta combinación específica de factores se da en la ciudad de Turrialba, donde un importante asentamiento humano se ha desarrollado y se sigue desarrollando, en un área naturalmente afectada por inundaciones, erosión fluvial, deslizamientos, terremotos y actividad volcánica.

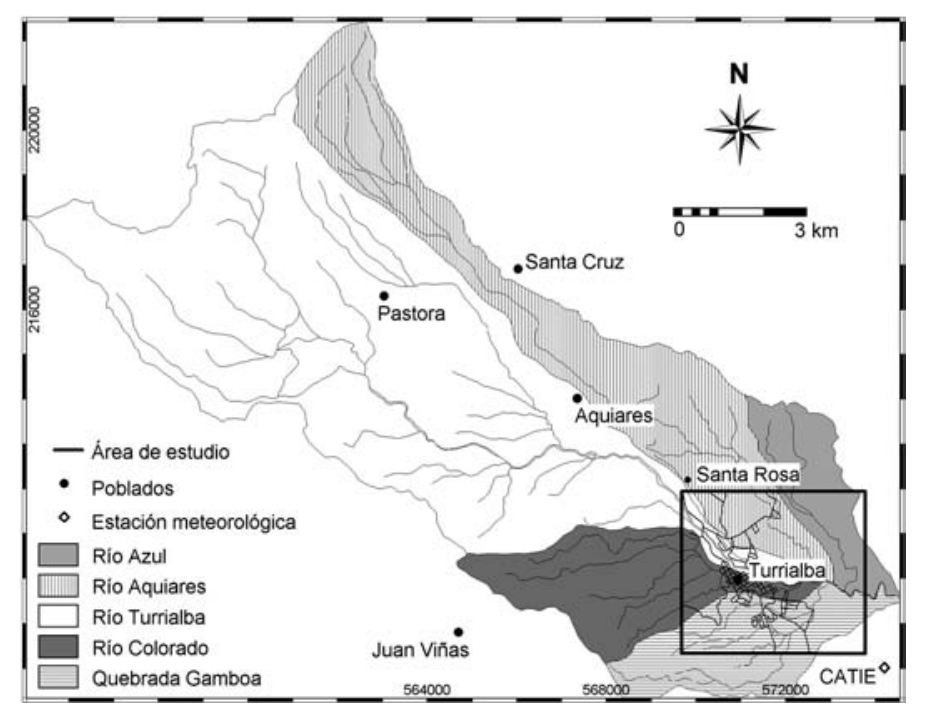

Fig. 1: Subdivisión de la cuenca del río Turrialba y definición del área de estudio dentro de ella. Se muestra también la ubicación de los principales poblados y de la estación meteorológica CATIE. 


\section{Antecedentes}

Tradicionalmente, las evaluaciones de amenaza de inundación se han llevado a cabo mediante análisis hidrológicos y modelaciones hidráulicas a partir de datos de caudales de los ríos responsables de las inundaciones. Lamentablemente, en el caso de la ciudad de Turrialba no fue posible llevar a cabo un análisis hidrológico debido a que únicamente existe información de caudales del río Turrialba, no de los ríos de menores dimensiones, los cuales son los causantes de las inundaciones (Colorado, Gamboa, Túnel y Barahona). La ausencia de levantamientos topográficos detallados de los valles de estos pequeños ríos imposibilitó también una modelación hidráulica.

Esta situación, observable no sólo en Turrialba sino en innumerables localidades, llevó a buscar una forma alternativa de evaluar la amenaza de inundación de la ciudad de Turrialba. Se pensó entonces en realizar una zonificación de amenaza con base en la ocurrencia y distribución de eventos históricos en el área. Debido a esto, se buscó recopilar, mediante entrevistas en el campo y revisión bibliográfica, tanta información como fuera posible, relacionada con diferentes inundaciones históricas. Desgraciadamente, la información encontrada en estudios anteriores es insuficiente y poco detallada, especialmente en lo que respecta a dimensiones del área inundada. Además, los pobladores de Turrialba no recuerdan las fechas de los eventos o incluso su ocurrencia misma, ya no están más en capacidad de dar detalles específicos sobre ellos o simplemente se acaban de mudar a la zona, así que desconocen todo acerca de los problemas de inundación.

La mayoría de los vecinos sólo recuerdan la última inundación de importancia que afectó la ciudad en febrero de 1996. Por esta razón, se buscó estudiar detalladamente este evento, para así extraer de él la mayor cantidad de información posible.

\section{Enfoque propuesto}

Considerando estos antecedentes, se decidió evaluar la amenaza de inundación en Turrialba mediante un enfoque basado en las características geomorfológicas y urbanas del área,

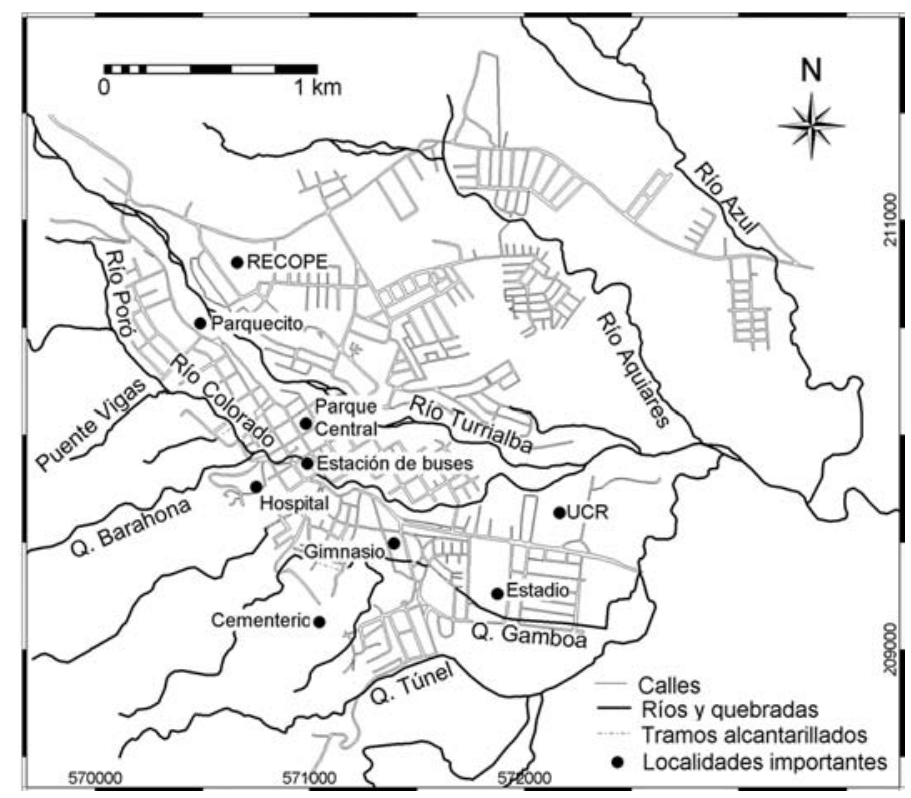

Fig. 2: Mapa de los ríos incluidos dentro del área de estudio. Se hace una diferenciación entre ríos, quebradas y tramos alcantarillados. También se indican algunas localidades a las que se hace referencia en el texto. 
complementadas con la información obtenida sobre el evento de 1996. La zonificación de amenaza estará basada entonces en una combinación de observaciones de campo y percepción remota.

El método seguido puede resumirse en las siguientes etapas:

Descripción geomorfológica del área de estudio, realizada a partir de fotointerpretación y observaciones de campo.

Determinación de las causas de inundación en la ciudad de Turrialba, mediante observaciones de campo, entrevistas a los afectados, revisión bibliográfica y fotointerpretación geomorfológica.

- Mapeo detallado, mediante entrevistas casa por casa, de la extensión y profundidades de la inundación de febrero de 1996. Además, identificación de los ríos que ocasionaron esta inundación y de los puntos a partir de los cuales se dio desbordamiento de las aguas.
Análisis estadístico de los registros de precipitaciones disponibles y determinación de sus períodos de retorno.

- Zonificación de amenaza de inundación para el área de estudio, a partir de la integración de toda la información recopilada.

\section{GEOMORFOLOGÍA DEL ÁREA DE ESTUDIO}

Se identificaron seis unidades geomorfológicas principales (Fig. 3), a partir de observaciones de campo y en especial, de interpretación de fotografías aéreas a diferentes escalas y fechas y de una imagen tipo anaglifo. Las unidades son:

Colinas denudacionales: localizadas en las esquinas SW y NE del área de estudio. Consisten de laderas de pendientes muy pronunciadas (30-60\%, de acuerdo con Calvo (1993)). Su litología está constituida por lavas, piroclastos y

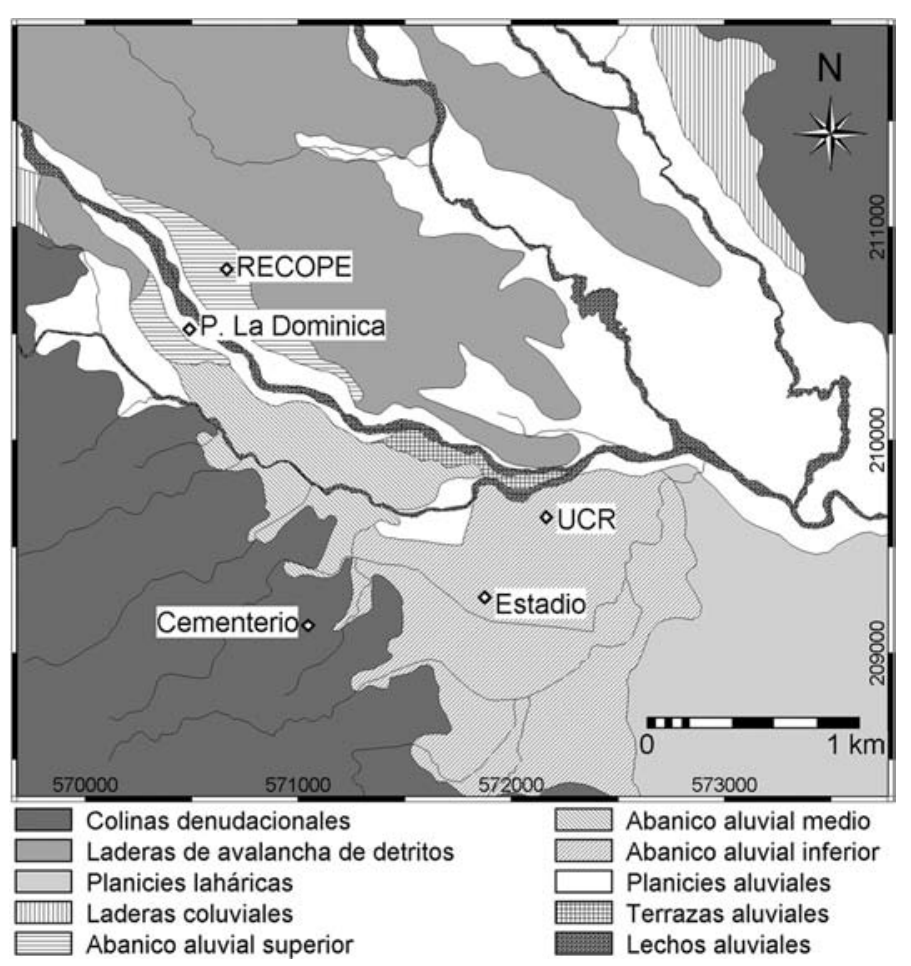

Fig. 3: Mapa geomorfológico del área de estudio. 
rocas sedimentarias, todas fuertemente afectadas por incisión de ríos y movimientos en masa. Estas colinas han sido intensamente meteorizadas, dando origen a una gruesa capa de suelos finos.

Laderas de debris avalanche: presentes en las partes central-norte y noroeste del área. Esta unidad se caracteriza por una topografía irregular con pendientes que van desde $3 \%$ hasta $20 \%$ (Calvo, 1993) y numerosas colinas redondeadas separadas por depresiones. Inmensos bloques de lava forman estas colinas. Otra característica importante es la ausencia de drenaje superficial, debido a la gran cantidad de fracturas que permiten al agua correr subterráneamente. La cobertura de suelo en esta unidad es escasa y de textura gruesa.

Planicies laháricas: se encuentran sólo en el sector sureste. Están representadas por un área plana (pendientes entre 1-3\%) y regular, con drenaje muy deficiente. Por lo tanto, el patrón de drenaje no está bien definido y es constantemente modificado por la actividad humana. Están formadas por materiales de origen volcánico y granulometría de fina a media.

Laderas coluviales: presentes en algunos sectores al pie de las colinas denudacionales, predominantemente en la esquina noreste del área de estudio. Esta unidad muestra pendientes de $10-20 \%$ y un sistema de drenaje moderadamente desarrollado. Están formadas por materiales gruesos provenientes de las colinas, con un desarrollo pobre del perfil de suelo.

Abanico aluvial: cubre las partes central y central-sur del área y fue formado por la depositación de materiales provenientes en su mayoría de los ríos Turrialba y Colorado, pero también de la quebrada Gamboa y sus afluentes. Este abanico está actualmente expuesto a una intensa erosión vertical y lateral, principalmente causada por los ríos Turrialba y Colorado. Se puede dividir en tres partes: abanicos superior, medio e inferior, diferenciables con base en el tamaño del grano y la pendiente.
Abanico superior: se caracteriza por pendientes de aproximadamente $10-15 \%$ y materiales gruesos a muy gruesos (textura blocosa).

Abanico medio: con pendientes de 5-10\% y materiales de grano medio a grueso (arenas, gravas y bloques). Las condiciones originales de buen drenaje de los abanicos superior y medio, debidas a su textura gruesa, han sido alteradas por el desarrollo urbano sobre ellos.

Abanico inferior: es casi plano (pendientes de 3-5\%), con materiales de grano medio a fino (arenas y limos) de baja permeabilidad. Representa una transición de la pendiente hacia los depósitos de lahar. La combinación de estos dos factores (pendiente y permeabilidad) causa que el drenaje en esta unidad sea deficiente.

Planicies aluviales: delgadas franjas de terreno a lo largo de los ríos principales, las cuales confluyen en una sola unidad cerca de la unión de estos. Pequeñas terrazas no mapeables separadamente por razones de escala, son incluidas dentro de esta forma de terreno. Estas planicies son zonas de composición heterogénea, con materiales que varían de gravas a limos. Hay sectores afectados por erosión lateral de las riberas de los ríos y son periódicamente inundadas.

En general, los ríos dentro del área de estudio son típicos de un sistema fluvio-erosivo. Tienen canales rectos y rocosos que bajan desde las colinas denudacionales, por los que el agua corre a grandes velocidades y con gran energía.

\section{PROBLEMÁTICA DE INUNDACIONES EN TURRIALBA Y SUS CAUSAS}

La ciudad de Turrialba es frecuentemente afectada por el desbordamiento de los ríos más pequeños que corren a través de ella (río Colorado, quebradas Gamboa, Poró y Túnel y otros pequeños cursos de agua). De acuerdo con datos históricos obtenidos de diversas fuentes (García, 1990; Zúñiga \& Arce, 1990; Aparicio, 1999; 
Cardona et al., 2000), los grandes ríos (Turrialba, Aquiares y Azul) no han causado inundaciones, al menos desde 1891. Solís et al. (1993) proponen que esto se debe principalmente a dos razones: primero, estos ríos tienen más capacidad de descarga y segundo, sus áreas de recarga se localizan en la cuenca superior del río Turrialba, mientras que las lluvias ocurren principalmente en las cuencas media e inferior. Según estos mismos autores, el río Turrialba puede soportar caudales pico con un período de retorno de 100 años sin desbordarse.

Los problemas de inundación en Turrialba no tienen solo causas naturales, sino que también son debidos a factores relacionados con la ubicación de los asentamientos humanos y su comportamiento. Se identificaron al menos tres tipos diferentes de causas de inundación para el área de estudio: meteorológicas, geomorfológicas y urbanas. Aparicio (1999) considera la precipitación como la principal causa de inundación en Turrialba, junto con la expansión urbana en áreas amenazadas.

\section{Causas meteorológicas}

El clima en la cuenca del río Turrialba es muy complejo, porque es influenciado por variables microclimáticas. Las condiciones meteorológicas en el área inducen lluvias torrenciales causadas por los vientos húmedos, procedentes del mar Caribe a través del valle del río Reventazón (Calvo, 1993). Una vez que los vientos alcanzan la cuenca del Turrialba, pueden seguir dos caminos diferentes: ingresan a la parte norte de la cuenca o continúan un poco más a lo largo del Reventazón y entran a ésta más hacia el sur.

Si los vientos van al norte, llueve principalmente en Aquiares y alrededores. En este caso, las aguas son drenadas directamente al río Turrialba, causando caudales pico en él. Si la humedad ingresa por el sur, la precipitación toma lugar especialmente sobre la cuenca del río Colorado. Entonces, caudales pico pueden causar desbordamiento de este río. Finalmente, cuando las masas de aire son suficientemente grandes, las lluvias extremas pueden alcanzar incluso la cuenca superior del Turrialba, produciéndose eventos catastróficos y no eventos moderados, como en los casos anteriores (García, 1990).

Los caudales de los ríos Colorado y Turrialba tienden a comportarse de acuerdo con este régimen pluviométrico dominado por factores microclimáticos. Estas descargas se caracterizan por muy bajos promedios pero con ocasionales eventos extremos (García, 1990). No se ha encontrado relación entre la ocurrencia de inundaciones en Turrialba y de eventos meteorológicos regionales como grandes tormentas tropicales, huracanes y depresiones tropicales (Aparicio, 1999).

Diciembre es el mes de mayor amenaza para la ciudad de Turrialba. Esto se debe a que éste, es el mes con la precipitación total mensual más alta, con los máximos caudales pico y también cuando la mayor cantidad de inundaciones ha ocurrido (Cuadro 2) (Aparicio, 1999). Esta situación puede explicarse desde un punto de vista meteorológico. Durante este mes, la nubosidad se localiza principalmente entre los 1200 y 1500 msnm, lo que corresponde con la cuenca superior del río Colorado, incrementándose las precipitaciones en esta área. Además, debido al efecto Foehn, una zona de altas precipitaciones se da entre los 600 y $1200 \mathrm{msnm}$, cubriéndose de esta manera la cuenca del Colorado casi en su totalidad (García, 1990).

Febrero, a pesar de ser un mes seco, también sobresale por la ocurrencia de importantes

\section{Cuadro 2}

Precipitaciones promedio de 2 días para los eventos del período 1949-1996 (Aparicio, 1999)

\begin{tabular}{llllr}
\hline Año & Mes & Fechas & $\begin{array}{l}\text { Precipitación } \\
\text { diaria }(\mathrm{mm})\end{array}$ & $\begin{array}{r}\text { Precipitac. } \\
\text { promedio de } \\
\text { 2 días (mm) }\end{array}$ \\
\hline 1949 & Diciembre & 3 y 4 & 64,5 y 288,3 & 176,4 \\
1966 & Febrero & 24 y 25 & 247,5 y 119,8 & 183,7 \\
1970 & Diciembre & 3 y 4 & 248,8 y 107,1 & 178,0 \\
1987 & Diciembre & 20 y 21 & 120,3 y 67,0 & 93,7 \\
1991 & Agosto & 11 y 12 & 118,1 y 120,4 & 119,3 \\
1996 & Febrero & 12 y 13 & 170,0 y 314,0 & 242,0 \\
\hline
\end{tabular}


caudales pico y altas precipitaciones y porque sigue a diciembre en el número de inundaciones (Cuadro 2) (Aparicio, 1999). Pero en este caso, ninguna explicación satisfactoria se ha encontrado. Posiblemente, estas inundaciones han sido causadas por lluvias torrenciales aisladas o por tormentas con ciertas características de intensidad o dirección.

\section{Causas geomorfológicas}

La ubicación de la ciudad de Turrialba en la parte baja de la cuenca del río del mismo nombre (Fig. 1) ha contribuido con los problemas de inundación en la zona. La ciudad está construida justo al pie de las pronunciadas laderas volcánicas, sobre los depósitos de abanico aluvial (Fig. 3), donde hay un abrupto cambio en las pendientes. Por lo tanto, los ríos bajan de las montañas a altas velocidades y con un gran potencial erosivo. La ciudad cubre una gran parte del abanico aluvial y de las planicies aluviales, y algunas veces incluso los lechos y bancos de los ríos, zonas que son naturalmente inundadas durante las crecidas.

Esta situación se ve agravada por la presencia de algunas curvas cerradas en los cauces y de cargas sedimentarias muy altas debidas a la ocurrencia de deslizamientos en las partes altas durante las lluvias (Madrigal, 1996).

La litología juega también un papel importante. Los ríos que causan las inundaciones provienen de montañas formadas por lavas muy meteorizadas. Esas lavas están cubiertas por una capa gruesa de suelos arcillosos, los cuales son muy impermeables. Debido a esto, la infiltración en estas montañas es muy reducida y una importante cantidad de lluvia es drenada a través de ríos de reducidas dimensiones.

\section{Causas urbanas}

Turrialba ha experimentado un gran crecimiento demográfico en las últimas décadas. Esta situación, combinada con erróneas políticas de planeamiento urbano y de uso de la tierra, ha intensificado los problemas de inundación.
En 1918, el distrito de Turrialba tenía una población de 5391 habitantes (Aparicio, 1999), mientras que en 2000 contaba ya con 32004 (INEC, 2001). A nivel de cantón, la población aumentó de 9216 habitantes en 1918 (Aparicio, 1999) a 68510 en 2000 (INEC, 2001). El distrito de Turrialba es ahora el más poblado del cantón, con un $46,7 \%$ de la población.

Pero el problema no es sólo que la ciudad ha crecido, sino que lo ha hecho expandiéndose a zonas con una gran dinámica hídrica en las planicies aluviales. Además, la extensa cobertura asfáltica ha reducido las zonas de infiltración a algunos parques y zonas verdes dispersos dentro del casco urbano. Ahora, las aguas de escorrentía han tenido que invadir las calles de la ciudad en su camino hacia los ríos, en vez de infiltrarse dentro de los depósitos permeables de abanico aluvial, aumentando la posibilidad de daño a edificios e infraestructura.

Un análisis comparativo entre el crecimiento demográfico y el régimen de lluvias fue llevado a cabo por Aparicio (1999). Éste muestra que desde 1949 la cantidad de lluvia necesaria para causar una inundación ha ido decreciendo en general, de acuerdo con el crecimiento de la población. La única excepción que puede hacerse es el evento de 1996 (Cuadro 2), durante el cual se registró la máxima precipitación promedio de dos días en 50 años (242 mm). Pero es interesante señalar que sólo la mitad de esa cantidad habría sido suficiente para causar un desbordamiento de los ríos (la inundación de 1991 fue causada por una precipitación de sólo 120 mm) y que en 1923 se registró una precipitación similar, sin generación de inundación.

La precipitación necesaria para causar una inundación durante la primera mitad del siglo XX era de más o menos $180 \mathrm{~mm}$. Ahora se considera que una precipitación máxima diaria de $100 \mathrm{~mm}$ es suficiente. Además, un incremento en la frecuencia de los eventos fue detectado desde 1970.

Por último, cabe agregar que se ha dado un cambio en el uso del suelo, de bosques a pastos o campos agrícolas, especialmente en la parte alta de la cuenca del río Turrialba. Esto ha llevado a un incremento en los caudales pico, la erosión, contaminación y a una disminución en la fertilidad de los suelos. 
PROBLEMÁTICA DE EROSIÓN FLUVIAL

La intensa erosión lateral ha sido un problema latente a lo largo de los mayores ríos del área de estudio, principalmente el Turrialba y el Colorado. Esta situación representa un gran peligro para la comunidad, no sólo por las casas e infraestructura que han sido y pueden llegar a ser destruidas o dañadas durante una crecida, sino también porque la erosión de los bancos de los ríos también representa un incremento en las posibilidades de que éste se desborde en ciertos puntos.

Esta situación es la que actualmente se está viviendo a causa de la erosión a lo largo del río Turrialba. No sólo numerosas casas y puentes han sido afectados, sino que ahora la ciudad se enfrenta con el peligro de que este río ha llegado a niveles de casi desbordamiento en los sitios en donde la erosión ha sido más intensa.

El río Turrialba tiene un cauce muy dinámico, que experimenta frecuentes cambios verticales y horizontales, de acuerdo con su necesidad de adaptar su perfil de equilibrio a las condiciones hidrológicas de la cuenca. El sector más peligroso y cambiante del río es donde cruza la ciudad. Los cambios horizontales se relacionan con variaciones en las curvas del río y los verticales con cambios en el micro-relieve del cauce. Es decir, que hay una interesante relación entre la morfología del cauce y el comportamiento del río durante una determinada descarga pico. Un mismo caudal pico puede ocasionar un desbordamiento en ciertas ocasiones, mientras que bajo otras condiciones no, todo depende de las condiciones del lecho y la carga de sedimentos en ese momento.

El problema ha llegado a tal punto que, en un lapso de poco más de un año, cuatro crecidas del río Turrialba causaron innumerables daños en puentes y viviendas. La primera de ellas fue el 29 de junio de 2001, la segunda en diciembre de ese mismo año, la tercera y más desastrosa de ellas, el 5 de mayo de 2002 y por último, el 1 de diciembre de 2002. Es posible observar por lo tanto, que las crecidas son cada vez más frecuentes y dañinas. Esto puede deberse al decrecimiento de la cobertura boscosa en la cuenca superior y el consecuente aumento de la erosión.
Entre más sedimento arrastre el río, más capacidad destructora adquiere éste. Otra posibilidad es que algún factor externo, como trabajos en el cauce, haya alterado el equilibrio del río Turrialba, haciendo que éste, en lugar de erosionar verticalmente, como predominantemente lo hacía, ahora tienda a depositar su carga a la altura del centro de Turrialba y a ampliar su cauce, erosionando lateralmente.

Numerosas pérdidas materiales y la urgente necesidad de tomar medidas estructurales y no-estructurales al respecto, han sido las consecuencias directas de este fenómeno.

\section{LA INUNDACIÓN DEL 14 DE FEBRERO DE 1996}

Como se explicó anteriormente, la información recolectada durante el trabajo de campo realizado entre junio y julio de 2001 se debió limitar a la inundación de febrero de 1996 y el área abarcada corresponde fundamentalmente con la zona inundada en esa ocasión (Fig. 4).

La información recopilada incluye las dimensiones del área inundada (Fig. 4) y las variaciones en la profundidad del agua dentro del área (Fig. 5). Además, se determinó la presencia y exacta ubicación de "cuellos de botella", áreas críticas (Fig. 4) y elementos de micro-relieve o urbanos que influyeron, de una manera u otra, en la distribución y el comportamiento del agua durante esta inundación.

Como cuello de botella se entiende una estructura (alcantarilla, puente) localizada en el cauce del río, la cual tiene dimensiones insuficientes en comparación con la capacidad de descarga del río, por lo que es frecuentemente sobrepasada por el agua y por lo tanto, actúa como punto de origen de una inundación. Una área crítica es también un punto de origen de inundación, pero corresponde con un sector en donde el desbordamiento es debido a factores naturales, como cambios de pendiente, convergencia de ríos o curvas cerradas en un río.

Un mapa de extensión y profundidades de la inundación de 1996 (Fig. 5) fue creado a partir de datos obtenidos por medio de entrevistas a 
los vecinos y respetando la distribución del agua que se consideró como lógica, de acuerdo con las condiciones de topografía y estructura urbana que se observaron en el campo.

El mapa de profundidades (Fig. 5) muestra que el agua cubrió la mayoría de los vecindarios localizados al sur del Río Turrialba, desde Pastor y Alto Cruz hasta Las Américas y Campabadal, incluyendo el centro de la ciudad (Fig. 6). Los niveles máximos de agua (entre 0,5-1 m) se alcanzaron en Las Américas, San Cayetano, La Guaria, Repasto y Mercado Municipal y la estación de buses en el centro (Fig. 2). El resto del área estuvo cubierta por menos de $50 \mathrm{~cm}$ de agua.

También se determinó que la distribución del agua dentro de la ciudad de Turrialba está principalmente determinada por la existencia, ubicación y comportamiento de los cuellos de botella y áreas críticas durante una específica descarga. Estos puntos se localizan a lo largo del río Colorado, las quebradas Gamboa y Túnel y algunos de sus afluentes (Fig. 4).

Esto significa que las inundaciones causadas por estos ríos tienden a tener siempre una distribución semejante, mostrando variantes básicamente sólo en las profundidades alcanzadas y leves cambios en la extensión del agua, dependiendo de la magnitud del caudal.

Una detallada descripción de lo que sucede en cada uno de estos puntos (basada principalmente en lo sucedido durante el evento de febrero de 1996) se da a continuación, con referencia a las figur. 6 y 2 ).

Punto 1: se localiza justo antes de la confluencia de los ríos Colorado y Poró. De acuerdo con Solís et al. (1994), durante eventos pico el río Poró arrastra enormes caudales, debido a la transición abrupta entre las áreas montañosas con pendientes de $9 \%$ y las planicies aluviales, con pendientes de $3 \%$ o menos. En esta área de transición el río deposita los materiales gruesos, construyendo un abanico aluvial cruzado por un canal poco definido. La insuficiente capacidad de descarga del río en este sector, junto con el giro abrupto que da antes de desembocar en el río Colorado, causan que el río Poró se desborde hacia su margen izquierda. Las calles de las

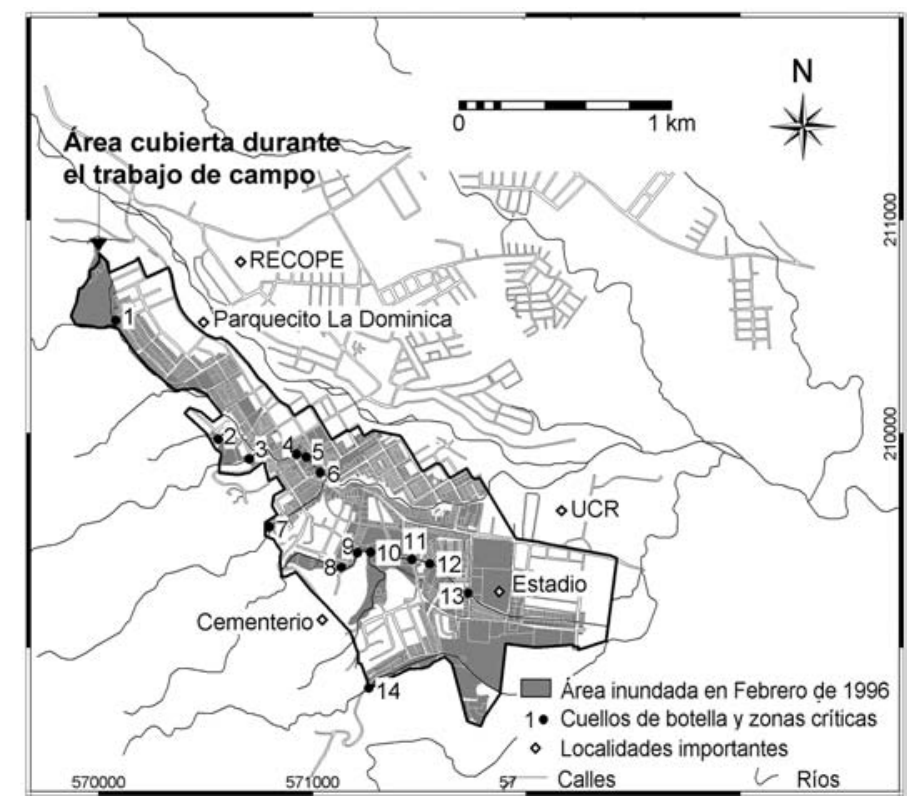

Fig. 4: Área cubierta durante el trabajo de campo. El mapa muestra también las áreas inundadas por el río Colorado, la quebrada Gamboa y sus afluentes, en febrero de 1996. Incluye también la localización de los cuellos de botella y áreas críticas identificados en el campo. 
áreas residenciales cercanas a este sitio (Alto Cruz y Pastor) son paralelas al río, así que actúan como canales secundarios por donde el agua corre en dirección a La Guaria, hasta alcanzar el centro de la ciudad.

Esta área crítica se encuentra en una zona plana que solía servir como reservorio natural temporal en caso de inundación. Pero la expansión urbana hacia este lado de la ciudad, así como algunos trabajos hechos por el dueño de la propiedad con el objetivo de evitar esta situación, han alterado esta condición natural (Solís et al., 1994).

Punto 2: en este punto, una pequeña quebrada que baja de las empinadas montañas alcanza la zona plana, donde se ubica una zona residencial. Pero el principal problema es que justo aquí, la quebrada es forzada a correr dentro de una alcantarilla de capacidad insuficiente. Por esta razón, la quebrada se desborda a partir de este cuello de botella, afectando las casas localizadas a lo largo de su camino hacia el río Colorado. La municipalidad ha llevado a cabo algunos trabajos en este sitio, pero aparentemente el problema aún no ha sido solucionado por completo.
Punto 3: corresponde con el puente sobre la quebrada Barahona, que se localiza justo antes de que ésta se una al río Colorado, a pocos metros de la carretera que lleva a San José. El cauce natural de esta quebrada ha sido estrechado por este pequeño puente, así como por una canalización de concreto que hay antes de él. Cuando un caudal pico alcanza este punto, arrastrando barro, basura y troncos, el área bajo el puente se ve bloqueada y la quebrada es obligada a desbordarse, afectando las casas, calles y negocios cercanos (Fig. 7).

Punto 4: localizado inmediatamente después del puente de la Calle 2, sobre el río Colorado. El ancho del cauce se ha visto reducido por la presencia de edificios y paredes de concreto en sus márgenes. En este punto además, el río gira a la derecha. Durante las crecidas, el nivel del río puede alcanzar y sobrepasar el muro de su margen izquierda y por consiguiente, el agua entra al Mercado Municipal (Fig. 7), causando graves daños, tal y como sucedió en 1996. Posteriormente, el agua sale del mercado por diferentes puntos, inundando los edificios y calles aledañas.

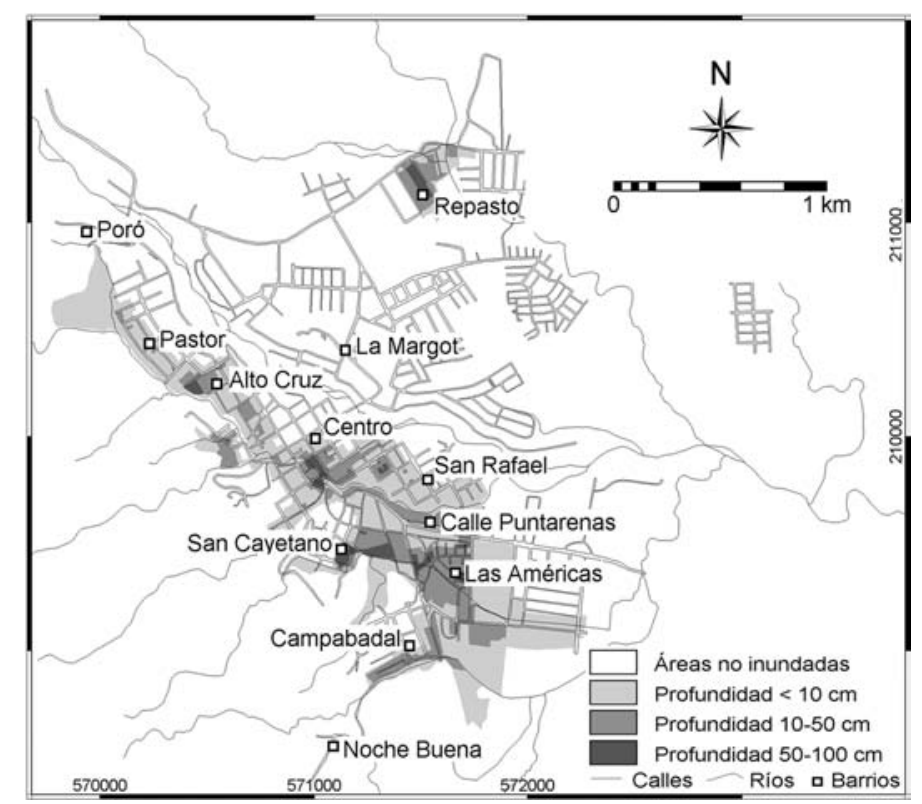

Fig. 5: Mapas de profundidades para la inundación de febrero de 1996 en la ciudad de Turrialba. 
Punto 5: una combinación de tres factores se da en este punto. Primero, el agua que había entrado al Mercado Municipal por el punto 4, sale de éste, a través de la entrada que se localiza aquí. Segundo, el río se desborda de nuevo al llegar al puente Independencia (Fig. 8). Finalmente, hay otro cuello de botella más porque el río Colorado es forzado a correr bajo la estación local de buses que está frente al mercado, a través de un túnel de reducidas dimensiones. Debido a todo esto, esta parte del centro de la ciudad puede considerarse uno de los sitios más críticos. Todas, las tiendas, el mercado, las estaciones de buses y gasolina y las calles son severamente afectadas por el agua lodosa cargada de basura que corre con gran energía. El nivel de agua durante una inundación en este sector puede alcanzar casi un metro.

Punto 6: corresponde con el final del túnel debajo de la estación de buses. El agua sale de este túnel con gran energía, desbordándose y erosionando intensamente sus márgenes. La inundación se propaga a partir de este punto en todas direcciones, pero especialmente hacia el noreste y este. Algunas carnicerías ubicadas aquí fueron completamente destruidas por el río Colorado en 1996 (Fig. 9).

Punto 7: cuello de botella generado por la entrada de una pequeña quebrada a una alcantarilla de dimensiones insuficientes ante un caudal pico. La quebrada se desborda, afectando las residencias circundantes.

Punto 8: la quebrada Gamboa corre a través de una estrecha alcantarilla, a lo largo de la parte trasera de una serie de casas. Durante descargas pico, la quebrada puede romper la canalización e ingresar en las viviendas. Posteriormente, sale por la parte delantera de ellas e inunda las calles, el parque y las casas vecinas. El nivel de

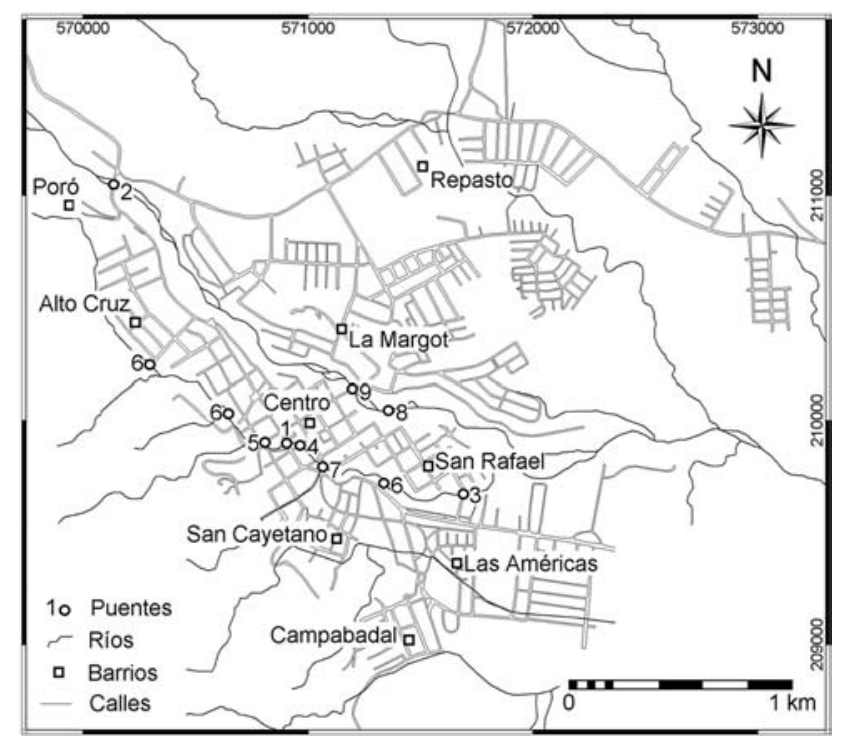

Fig. 6: Localización de puentes y barrios dentro del área de estudio. 1: Pte. de la Calle 2, 2: Pte. Alegría, 3: Pte. del Balneario, 4: Pte. Independencia, 5: Pte. Panamá, 6: Pte. Peatonal, 7: Pte. del Ferrocarril, 8: Pte. Negro, 9: Pte. Blanco. 
agua puede llegar a ser bastante alto, cercano a los $50 \mathrm{~cm}$, ya que no fluye fácilmente de vuelta a su cauce debido a la presencia de las casas.

Punto 9: una situación bastante similar a la descrita en el punto 8 se da lugar en este sitio. De nuevo, la quebrada Gamboa se desborda debido a las escasas dimensiones de otro alcantarillado e inunda las casas vecinas con hasta 50 $\mathrm{cm}$ de agua.

Punto 10: corresponde con la zona plana donde se ubican el Gimnasio Municipal y algunas bodegas y que es inundada por la quebrada Gamboa, en un proceso aparentemente natural. Algunos vecinos dicen que esta área solía ser un pantano, pero el crecimiento urbano lo hizo desaparecer.
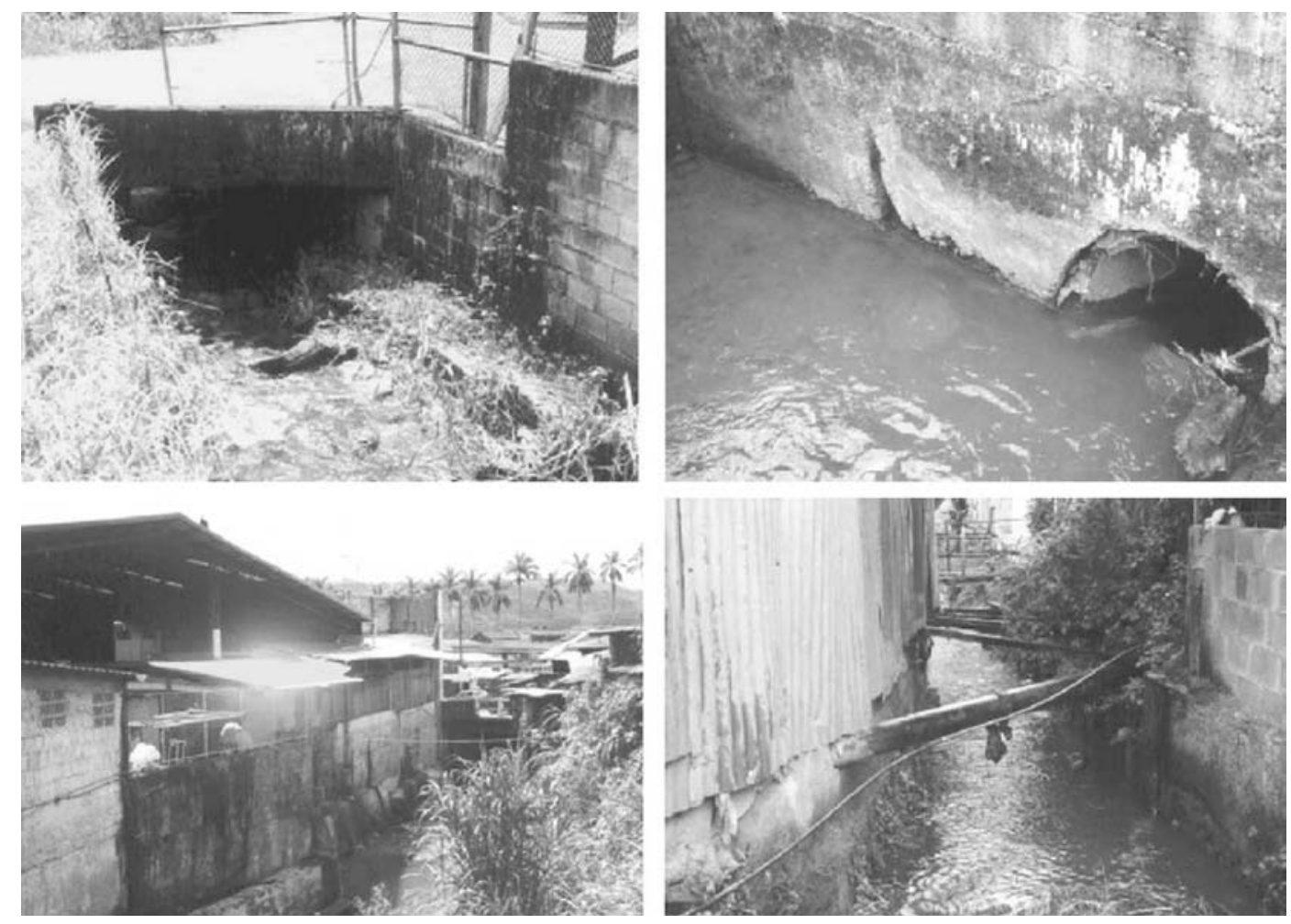

Fig. 7: Puntos críticos donde los ríos se desbordaron en febrero de 1996: Punto 3 (esquina superior izquierda), Punto 4 (esquina inferior izquierda), Punto 11 (esquina superior derecha) y tramo de la quebrada Gamboa justo antes del Punto 12 (esquina inferior derecha). 
profundidad del agua durante la inundación puede ser de más de $50 \mathrm{~cm}$, especialmente hacia el norte del Punto 13. Aquí, la presencia de una tapia provoca que el agua quede encerrada dentro de las alamedas.

Punto 14: comprende la parte suroeste de barrio Campabadal. Aquí, el cauce natural de la quebrada Túnel fue modificado debido al desarrollo urbano, cambiando sus dimensiones y recorrido. Debido a esto, la quebrada tiende a desbordarse durante las crecidas, inundando una extensa área en su camino a través de las zonas residenciales. En 1996, un flujo de lodo en esta quebrada destruyó varias casas en este sector.

\section{ANÁLISIS ESTADÍSTICO DE PRECIPITACIONES}

Con el propósito de obtener información relacionada con la cantidad de lluvia esperada para una determinada probabilidad de ocurrencia, se realizó un procesamiento estadístico sencillo. Para ello se contó con los datos de precipitaciones diarias máximas por año (Aparicio, 1999) registradas por la estación meteorológica CATIE para el período 1949 - 1998 (Cuadro 3). La validez de la utilización de estos datos como

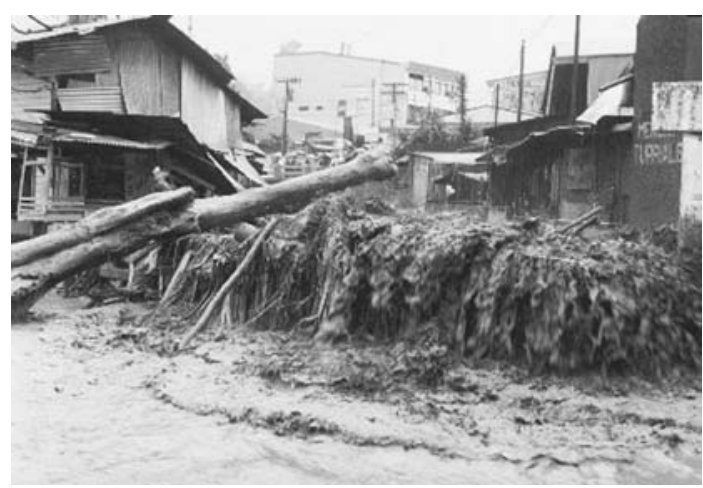

Fig. 8: Desbordamiento del río Colorado a la altura del Puente Independencia durante la inundación de febrero de 1996. método para obtener un parámetro de recurrencia para los eventos de inundación, se basa en el hecho de que todas las inundaciones ocurridas durante este mismo período (1949 - 98) se dieron precisamente bajo influencia de estas precipitaciones pico (Cuadro 2). Además, es posible observar que la precipitación diaria máxima registrada por la estación CATIE desde 1949 fue aquélla causante de la inundación de Febrero de 1996 (314 mm).

Para el cálculo de los períodos de retorno de las precipitaciones se siguió el método de Gumbel (Viessman et al., 1989), el cual se resume a continuación. Los resultados obtenidos a partir de su aplicación se muestran en el cuadro 4 y en la figura 10.

\section{Cuadro 3}

Registro de precipitaciones diarias máximas por año para el período 1949 - 1998, para la estación CATIE (Aparicio, 1999)

\begin{tabular}{|c|c|c|c|}
\hline Año & $\begin{array}{l}\text { Precipitación } \\
\quad(\mathrm{mm})\end{array}$ & Año & $\begin{array}{l}\text { Precipitación } \\
(\mathrm{mm})\end{array}$ \\
\hline 1949 & 288 & 1974 & 154 \\
\hline 1950 & 96 & 1975 & 152 \\
\hline 1951 & 104 & 1976 & 126 \\
\hline 1952 & 83 & 1977 & 84 \\
\hline 1953 & 140 & 1978 & 85 \\
\hline 1954 & 78 & 1979 & 65 \\
\hline 1955 & 109 & 1980 & 148 \\
\hline 1956 & 109 & 1981 & 150 \\
\hline 1957 & 106 & 1982 & 115 \\
\hline 1958 & 65 & 1983 & 107 \\
\hline 1959 & 102 & 1984 & 166 \\
\hline 1960 & 109 & 1985 & 91 \\
\hline 1961 & 85 & 1986 & 176 \\
\hline 1962 & 96 & 1987 & 120 \\
\hline 1963 & 110 & 1988 & 241 \\
\hline 1964 & 61 & 1989 & 100 \\
\hline 1965 & 82 & 1990 & 67 \\
\hline 1966 & 248 & 1991 & 120 \\
\hline 1967 & 102 & 1992 & 131 \\
\hline 1968 & 131 & 1993 & 220 \\
\hline 1969 & 107 & 1994 & 96 \\
\hline 1970 & 288 & 1995 & 78 \\
\hline 1971 & 86 & 1996 & 314 \\
\hline 1972 & 127 & 1997 & 235 \\
\hline 1973 & 124 & 1998 & 121 \\
\hline
\end{tabular}


Cuadro 4

Período de retorno de diferentes precipitaciones, obtenido mediante el método de Gumbel (Viessman et al., 1989)

\begin{tabular}{ccc}
\hline $\begin{array}{c}\text { Período de } \\
\text { retorno (años) }\end{array}$ & $\begin{array}{c}\text { Posición de } \\
\text { ploteo Y }(\mathrm{mm})\end{array}$ & Precipitación \\
\hline 50 & 3,9 & 300 \\
25 & 3,2 & 265 \\
10 & 2,3 & 220 \\
5 & 1,5 & 180 \\
\hline
\end{tabular}

- Los datos de precipitación se ordenan de menor a mayor.

- Se asigna a cada dato un valor J, en el intervalo de 1 a $n$ ( $\mathrm{n}=$ número total de datos), asignando el número 1 al valor menor y $\mathrm{n}$ al valor mayor.

- $\quad$ La probabilidad de no-excedencia se calcula con la fórmula $P=J /(n+1)$ y el período de retorno con la fórmula $\mathrm{R}=1 /(1-\mathrm{P})$

- Con el propósito de graficar los resultados, se calcula para cada dato una posición de ploteo $\mathrm{Y}(=-\ln (-\ln (\mathrm{P})))$, valor contra el cual se grafica cada precipitación. Posteriormente, se construye una línea de mejor ajuste (Fig. 10)

- A partir de este gráfico, puede leerse el valor Y para cualquier precipitación y su correspondiente período de retorno puede ser simplemente calculado con la fórmula $\mathrm{P}=\left(\mathrm{e}^{-\mathrm{e}}\right)^{-\mathrm{Y}}$. $\mathrm{P}$ es entonces sustituido en la fórmula de $\mathrm{R}$ dada anteriormente.

\section{MAPA DE AMENAZA DE INUNDACIÓN}

Este mapa fue el resultado de una combinación del mapa geomorfológico (Fig. 3) construido a partir principalmente de fotointerpretación a diferentes escalas y de las observaciones de campo realizadas. Un diferente grado de amenaza fue asignado a cada unidad geomorfológica (Fig. 11) con base en su ubicación, pendiente, patrón de drenaje, tamaño de grano, permeabilidad y situación durante la inundación de febrero de 1996.
La forma de algunas unidades fue modificada con base en condiciones locales que incrementan la susceptibilidad a inundación en un sector específico, tales como la presencia de cuellos de botella o puntos críticos.

Los períodos de recurrencia asignados son aproximaciones iniciales establecidas tomando como referencia los relatos de vecinos sobre la frecuencia en la ocurrencia de eventos en un determinado sector y el período de retorno de aproximadamente 50 años estimado anteriormente para la inundación de 1996 (314 mm), utilizando registros de precipitaciones de la estación CATIE. Cabe destacar que el uso de este método estadístico, basado en datos de precipitación, implica que estos períodos de retorno calculados son independientes de características propias de cada una de las sub-cuencas causantes de las inundaciones (pendiente, cobertura vegetal, etc.). Estas características son las responsables, en un evento real, de diferencias de respuesta o comportamiento entre las sub-cuencas, ante una determinada precipitación. Pero la consideración de todas estas variables va más allá del alcance de esta investigación.

Se establecieron cuatro categorías de amenaza de inundación (Fig. 11):

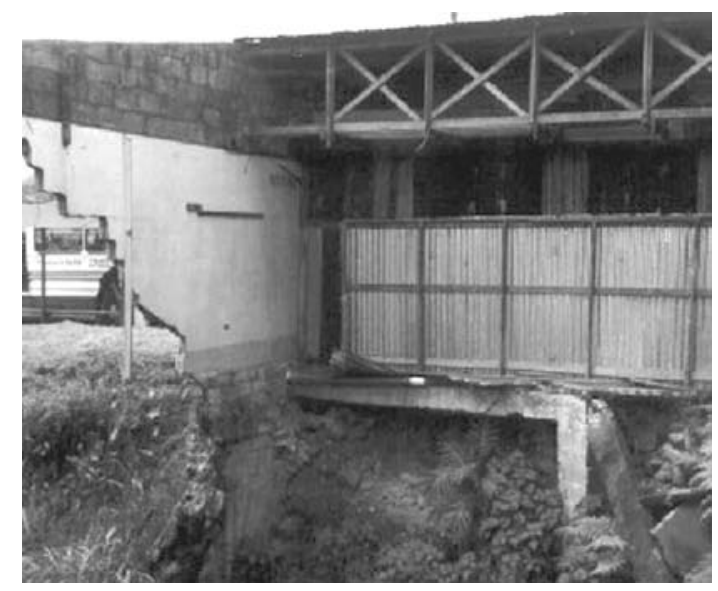

Fig. 9: Carnicería colapsada debido a la erosión ocasionada por el río Colorado durante la inundación de febrero de 1996. 
1. Áreas con alta amenaza de inundación (inundaciones de alta frecuencia, período de retorno menor a 25 años): localizadas principalmente en la parte media del abanico aluvial, la cual es frecuentemente inundada por el río Colorado y sus afluentes. Esta unidad geomorfológica tiene una pendiente de 5-10\% y

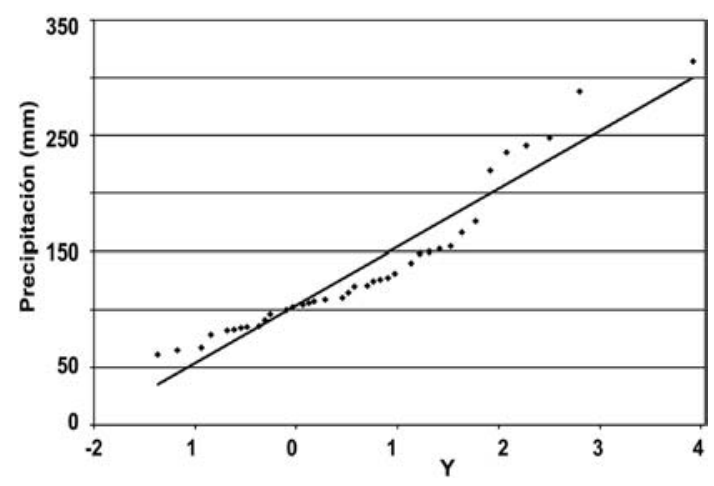

Fig. 10: Datos de precipitación contra posición de ploteo Y y su correspondiente línea de mejor ajuste. está formada por materiales permeables. Pero esta permeabilidad original se ha visto severamente disminuida por el desarrollo del centro de la ciudad sobre estos depósitos. La pequeña parte del abanico inferior y de las planicies aluviales incluida dentro de esta categoría está relacionada con la presencia de condiciones críticas en estos sectores.

2. Áreas con amenaza de inundación media (inundaciones de frecuencia intermedia, período de retorno de 25 a 75 años): corresponde con la mayor parte del abanico inferior y las planicies aluviales. Se podría pensar que la amenaza de inundación en el abanico inferior es mayor que en el abanico medio, por ser el primero más plano y menos permeable que el segundo. Pero el abanico inferior se localiza más lejos de los sectores críticos dentro de la ciudad, de los ríos que causan las inundaciones. Por esta razón su grado de amenaza es más bajo. Las planicies aluviales incluidas dentro de esta unidad son

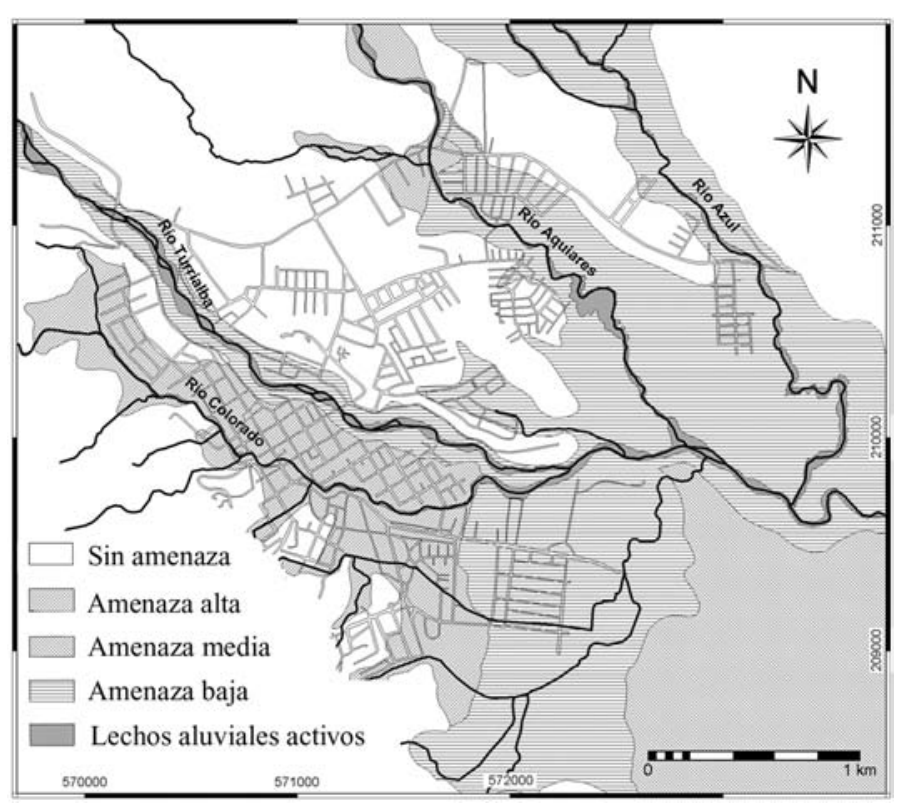

Fig. 11: Mapa de amenaza de inundación del área de estudio. 
aquellas localizadas a lo largo de los ríos Turrialba, Aquiares y Azul. Estos ríos tienen valles profundos, lo que reduce la probabilidad de desbordamiento.

\section{3. Áreas con baja amenaza de inundación} (inundaciones de baja frecuencia, período de retorno mayor a 75 años): las planicies laháricas ubicadas en la esquina sureste del área de estudio son las únicas áreas incluidas dentro de esta unidad. Estas planicies son planas y tienen un drenaje natural deficiente, pero se encuentran localizadas lejos de las fuentes de inundación. No hay reporte de inundaciones históricas en este sector.

4. Áreas sin amenaza de inundación: colinas denudacionales, laderas de debris avalanche y abanico superior son las unidades consideradas dentro de la categoría de no amenazadas por inundaciones. Por el contrario, sus pronunciadas pendientes las convierten en óptimas zonas para la concentración de las aguas de escorrentía que podrían causar inundaciones en las zonas bajas.

\section{CONCLUSIONES}

Aunque las inundaciones en Turrialba están relacionadas con lluvias torrenciales y locales, su ocurrencia depende también de factores antrópicos, tales como la ubicación desordenada de los asentamientos humanos y su rápido desarrollo, combinado con incorrectas políticas urbanas y de uso del suelo.

Tradicionalmente, las inundaciones han sido ocasionadas por los ríos pequeños y los graves problemas de erosión se han dado a lo largo de los ríos grandes. Pero actualmente el río Turrialba está al borde de convertirse también en una amenaza de inundación, debido al debilitamiento de sus márgenes durante sus últimos períodos de erosión intensa.

La distribución (extensión y profundidad) del agua dentro de la ciudad durante las inundaciones está principalmente determinada por la ubicación de los cuellos de botella a lo largo de los ríos.

Las áreas con mayor amenaza de inundación corresponden principalmente con la parte media del abanico aluvial, el cual por sus características de permeabilidad y pendiente no debería ser tan frecuentemente inundado, pero lo es. Esto indica que el crecimiento urbano sobre esta área ha incrementado la probabilidad de inundación, al aumentarse la escorrentía. Por el contrario, en las áreas con amenaza de inundación moderada o baja (áreas con menos desarrollo urbanístico) las inundaciones se dan de una manera más natural y menos violenta, debido a la presencia de áreas planas con drenaje deficiente y baja permeabilidad.

Podría entonces afirmarse no sólo que la ciudad de Turrialba se encuentra en el área de mayor amenaza de inundación, sino también que esta alta amenaza es una consecuencia directa de la presencia de la ciudad en esta área.

\section{RECOMENDACIONES}

La zonación de amenaza de inundación llevada a cabo podría enriquecerse a partir de un trabajo de campo más intenso enfocado a un mapeo geomorfológico de más detalle, que sirva como complemento a la fotointerpretación. Sería también de gran valor la incorporación de información relacionada con geología, suelos y características de estos como granulometría y permeabilidad.

El monitoreo de caudales en los ríos y quebradas causantes de inundaciones vendría a aportar información invaluable relacionada con caudales pico generadores de inundaciones. A partir de estos caudales se determinarían períodos de retorno y se seleccionarían caudales de diseño para ser utilizados a la hora de rediseñar los puentes y otras estructuras que actúan como cuellos de botella.

Llevar a cabo, por parte de las autoridades locales, una campaña de información y concientización de la población, como una medida de mitigación ante la problemática de inundaciones existente en la comunidad. 


\section{REFERENCIAS}

ALEXANDER, D., 1993: Natural disasters. 632 págs. Routledge, Londres.

APARICIO, M.J., 1999: Dinámica de inundaciones del río Colorado e impacto en Turrialba, Costa Rica. - 79 págs. Centro Agron. Trop. Invest. y Enseñanza, Turrialba [Tesis M.Sc.].

BADILLA, E., 2002: Flood hazard, vulnerability and risk assessment in the city of Turrialba, Costa Rica. - 82 págs. Internat. Inst. Geo-Information Science and Earth Observation, Enschede, Holanda [Tesis M.Sc.].

CALVO, C. A., 1993: Dinámica, uso apropiado y sostenible de la tierra, en la cuenca del río Turrialba. - 186 págs. Centro Agron. Trop. Invest. y Enseñanza, Turrialba [Tesis M.Sc.].

CARDONA, M., CALZADILLA, M., SÁNCHEZ, P., BARILLAS, M., LIRA, E., RODRÍGUEZ, M., MOLINA, G., DERAS, J., RIVERA, J., FÚNES, J., CRUZ, E., JARQUÍN, I., PÉREZ, C., SALGADO, D., BARRANTES, S., CLÍMENT, Á., ORTEGA, M. \& RIVERA, L., 2000: Estudio integral de amenazas naturales en la cuenca del río Turrialba, cantón de Turrialba, República de Costa Rica, Centroamérica. - 62 págs. Internat. Inst. Aerospace Survey and Earth Sciences, Enschede, Holanda [Informe interno].

GARCÍA, J. D., 1990: El análisis de cuencas hidrográficas aplicado al problema de inundaciones. El caso de la Ciudad de Turrialba. - 183 págs. Escuela Historia y Geografía, Depto. Geografía, Univ. Costa Rica, San José [Tesis de grado].
INEC, 2001: IX Censo nacional de población y V de vivienda, Costa Rica: cifras preliminares por provincia, cantón y distrito. - 32 págs. Inst. Nac. Estadísticas y Censos, San José.

MADRIGAL, J.E., 1996: Problemática por deslizamientos e inundaciones en el cantón de Turrialba y Jiménez. - 8 págs. Com. Nac. de Emergencia, San José [Informe interno].

SOLÍS, H., BEAULIEU, N., \& CHACÓN, J. J., 1994: Análisis del problema de inundaciones en el río Colorado, Turrialba, Costa Rica. - 23 págs. CATIE, JASEC, MOPT, San José [Informe técnico].

SOLÍS, H., OREAMUNO, R., MURILLO, W. \& CHACÓN, J. J., 1993: Modelación hidrológica e hidráulica para el control de inundaciones en Centroamérica. Casos ríos Purires y Turrialba. - 61 págs. Centro Agron. Trop. Invest. y Enseñanza, Turrialba [Informe técnico].

UNDRO, 1983: Space applications for the acquisition and dissemination of disasterrelated data. - Oficina de las Naciones Unidas para la coordinación de la atención de desastres, Expert Meeting, Ginebra, 1-71.

VIESSMAN, W., LEWIS, G.L. \& KNAPP, J.W., 1989: Introduction to Hydrology.- 325 págs. Harper and Row Publ. New York.

ZÚÑIGA, L.I. \& ARCE, J.R., 1990: Problemática de las inundaciones en Costa Rica: una propuesta de análisis. - 135 págs. Escuela Ingeniería Civil, Univ. Costa Rica, San José [Tesis de grado]. 
Fé de erratas: Fig.11, pág. 106

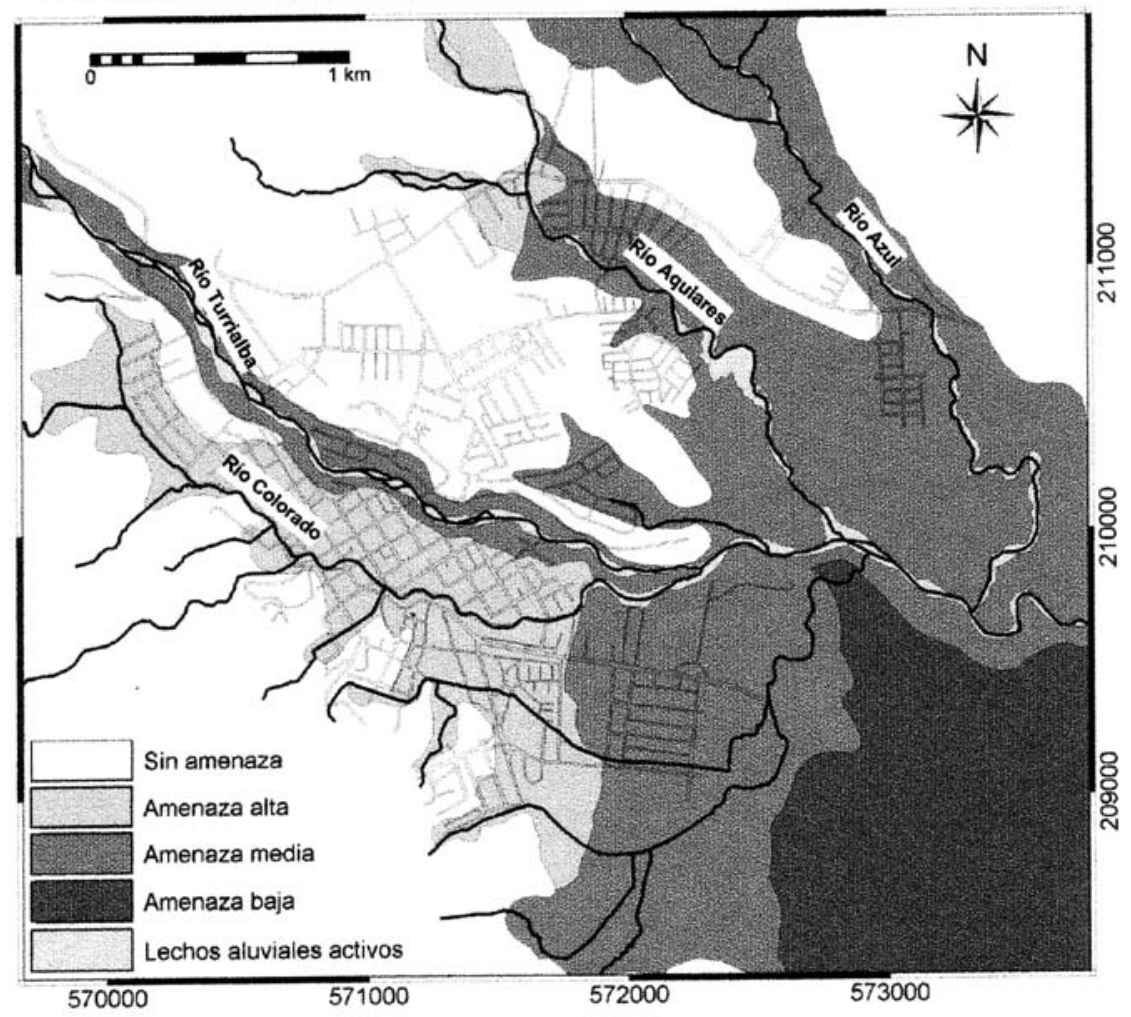

Fig.11: Mapa de amenaza de inundación del área de estudio 